\title{
Study on Surface Defect Detection of Automobile Seat
}

\author{
X.M. Zhang, H. Wang \\ Shenyang University of Technology \\ Shenyang
}

\begin{abstract}
For scratches and other defects may be generated in the vehicle seat production process, an on-line detection method is introduced based on machine vision. The defects are extracted by graying, image filtering and image segmentation based on $\mathrm{K}$ means clustering region growing algorithm. The illustration shows good effect of presented method in defect. The method used in this paper successfully realizes the detection of uneven texture surface.
\end{abstract}

Keywords: machine vision; image processing; region growing algorithm; quality inspection

\section{INTRODUCTION}

Machine vision is a comprehensive application of computer technology to simulate the human visual function, instead of eyes to extract information from the target in the image of a new detection technique [1]. In recent years, the technology of machine vision has been widely used in many fields. Research on the application of Chang in the industrial field, J.Y. et al developed a machine vision guidance and positioning system which is used for flexible circuit board's assemble on the hard disk [2]. Al-kindi et al. applied the technology of machine vision to mechanical parts surface roughness in detection work [3]. Most of the current detection method for a particular area, types and parameters of defect detection mostly focus on the surface structure of uniform and easy to metal surface detection. But for such as leather which has uneven texture surface quality detection is difficult to achieve.

As an important part of automobile, the surface quality of car seat is influence car quality. This text is in the light of the seat surface quality detection problem of this car seat production line for a company in the introduction of machine vision, detection, in order to improve the production efficiency, guarantee the quality of the seat factory.

\section{THE DETECTED OBJECT DESCRIPTION}

Car seat is a reference measure of automotive grade. In the production process of seat, because the process of system vulnerabilities or other factors, the surface of the seat will have some defects, such as scratches, breakage, as shown in figure 1.

In addition to the seat production line the testing work is affected by noise, vibration and space, the design of efficient detection system has the quite great difficulty.

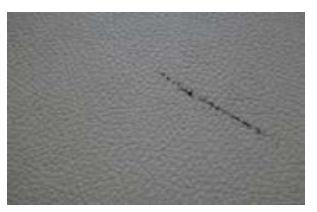

FIGURE I. THE SCRATCH DEFECTS OF SEAT SURFACE

\section{THE COMPOSITION AND PRINCIPLE OF DETECTION SYSTEM}

Detection system is mainly composed of image acquisition, processing and the output of the control computer, the specific structure as shown in figure 2[4]. Its working principle is: in a certain light conditions using CCD camera to acquire image information for seat surface, the CCD camera can turn real information of nature into digital image which can be identified by computer, and then processed and analysed and determine whether there are surface defects, if flawed, controlled by the system and remove the defects of defective products.

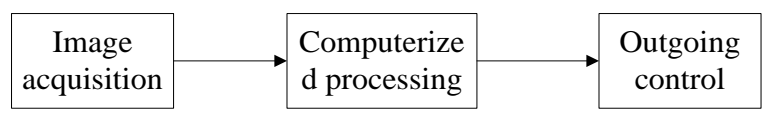

FIGURE II. THE COMPOSITION OF DETECTION SYSTEM.

Because of light changes in production environment, multiple mode of CCD camera is adopted in image acquisition scheme, in the light irradiation LED lamp, to highlight the seat surface defect feature. Digital image acquisition is input to the computer, and then processing operation of image pretreatment, image segmentation, feature extraction and a series of processing.

\section{THE PROCESSING OF SEAT IMAGE}

Image processing is done by computing the programming software, through the programming operation to pre-process, image segmentation, feature extraction arithmetic operation on the image acquisition.

\section{A. Seat Image Pre-processing}

Due to the characteristics of strong seismic large, storage capacity, high speed of industrial computer, using CCD camera which fixed production line stations that can obtain real-time image acquisition process, but in process of collecting the seat surface image, because of the influence of light source, vibration and other factors, it is inevitable that there will be a lot of noise in the image, we need to pre-process the digital image acquisition.

1) Gray-scale image processing: The image acquisition CCD camera is the digital image, the image model is RGB 
space model, namely R (Red), G (Green), B (Blue). RGB system is through change of the intensity ratios of $R, G, B$ to get different color.

$$
C=r R+g G+b B
$$

Wherein, C represents a certain color, R, G, B on behalf of tricolor, and R, G, B representing the proportion coefficient, and $r+b+g=1[5]$. In order to simplify the later operation process, need to gray image processing, generate a special image which $\mathrm{R}=\mathrm{G}=\mathrm{B}$, through the RGB three-dimensional space to onedimensional space mapping relationship between gray to gray image processing. Treatment results are shown in figure 3.

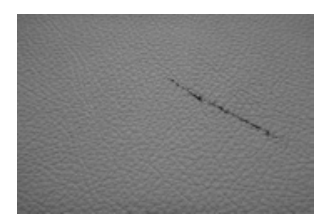

FIGURE III. GRAY IMAGE.

2) Image filter processing: Because of the influence of seat production line light source and vibration and other factors, the image collection, it is inevitable that there will be a lot of noise, so need to find a way which can eliminate the noise, but also can ensure the integrity of the filtering means the details of images.

Median filtering is a nonlinear filtering technique, it will be the gray value of each pixel which is set for all pixels of the points of a median neighborhood within the window, thereby eliminating the isolated noise points [6]. This method is essentially a two-dimensional array of filter, the filter window is two-dimensional. Two dimensional median filter output:

$$
g(x, y)=\operatorname{med}\{f(x-k, \quad y-1), \quad(k, 1 \in W)\}
$$

Among them, $\mathrm{f}(\mathrm{x}, \mathrm{y}), \mathrm{g}(\mathrm{x}, \mathrm{y})$ were the original image and the processing image. $\mathrm{W}$ is $2 \mathrm{D}$ template which is usually divided of $3 * 3,5 * 5,7 * 7$ region, also can be different shapes, such as linear, square, circular, the figure ten and the circular etc. This paper selects the input image which pixel is related to $3 * 3,5 * 5,7 * 7$. Results of seat surface are shown in figure 4 :

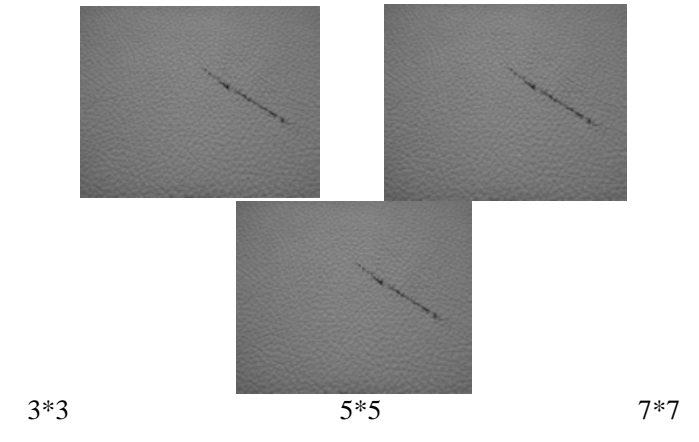

FIGURE IV. THE RESULTS AFTER MEDIAN FILTERING.

Fig. 4 shows the effect of different sizes of different selection filter. Sharpness of each image can calculate by the following formula which is also called image edge sharpness, comparing the three image clarity:

$$
A_{c}=\frac{B_{r}}{g_{s}}
$$

In the formula, Ac is the sharpness of vision; $\mathrm{Br}$ is the brightness changes at the transition zone on both sides of the position; gs is the change of the position at transition region.

According to the calculation, $3 * 3$ processing image sharpness is $0.693,5 * 5$ processing image sharpness is 0.677 , $7 * 7$ processing image sharpness is 0.66 . Thus, the highest resolution image window size of filter in the three images is $3 * 3$, filtering effect is the best, so the choice of image processing window of $3 * 3$ as the object of later image segmentation.

\section{B. Seat Image Segmentation}

1) The seat surface defect region extraction algorithm: Due to defects in the surface of the seat with no rules, uncertainty and other characteristics, some defect coverage area interconnected, so this is connected with central region growing method to expand the defect area. In the presence of major regional growth during the following questions [7]:

(1) Initialization seed region selection.

(2) Compare the gray-scale pixel with its neighboring pixels seed point, if the difference is less than a preset threshold value, it can be combined.

(3) Compare the average gray new merged area with its neighboring pixels, if the difference is less than a preset threshold value, it can be combined.

(4) Stopping regional growth when there is no point merge neighborhood.

(5) However, for selecting said initial seed pixel and the threshold directly affects the result of image segmentation, in general, need to be chosen with reference to specific problems [8].

2) Regional growth based on the image histogram to select the initial seed point: In K- clustering algorithms of traditional regional growth, $\mathrm{K}$ is given in advance, but it is difficult to choice, what works best is not clear segmentation, this thesis combining theory mixed ANOVA F statistic to determine the optimal number of categories. Infer whether they from the same set of by comparison with the threshold value of $\mathrm{F}$, its distribution shown in Figure 6.

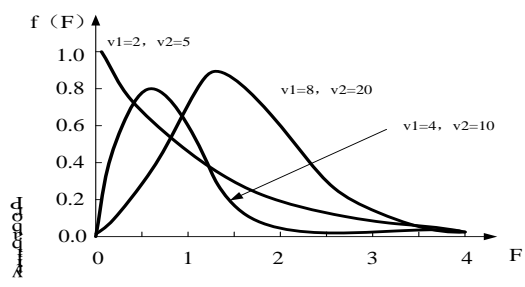

FIGURE V. VARIANCE PROBABILITY DISTRIBUTION.

The basic idea of analysis of variance [9]:

(1) Put all the data as several sets of data samples extracted with a collection, calculated the total variation: 


$$
S S_{T}=\sum_{1}^{K} \sum_{1}^{n}\left(X_{i j}-\bar{X}\right)^{2}
$$

(2) Analyze possible causes of variation, broke the total variation into the quadratic sum of the variation and degrees freedom based on these reasons;

(3) Do division between variance and error variance, get the $\mathrm{F}$ value;

(4) If $F>1$, there can be significant differences between the determination process, then continue to do multiple comparisons;

(5) If $\mathrm{F}$ is closed to 1 , it mean there was no significant difference between said handle, analysis is ending.

It can be calculated according to the method described above, the best classification clustering number of $\mathrm{K}$ is 3 , so we choice 3.

Since the defective area of the seat surface is lower than some other areas, we need to do a statistical about the gray value of each pixel in processed image, Generate the gray level histogram(Figure 7),distributed according to the situation

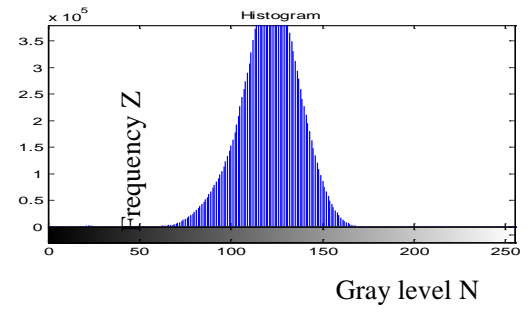

FIGURE VI. THE GRAY LEVEL HISTOGRAM OF SEATS.

of gray histogram, we can select the area of nuclear, select the gray value point which has low grayscale value and high probability as the regional growth of the initial seed point, Growth defects part of the image area by K- means clustering region growing method. The image after regional growth and corresponding gray value image as shown in Figure 8, 9.

After passing through the seat image segmentation defect image shown in Figure 8, we can clearly understand the difference between defect region and surrounding texture. Through the gray distribution of computer software statistical image, in Figure 9 of histogram, we can identify the highest frequency of gray level is 118 .

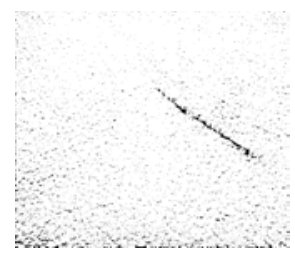

FIGURE VII. AFTER THE GROWTH OF CLUSTERIN.

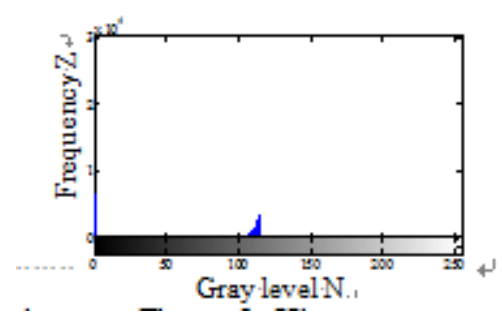

FIGURE VIII. HISTOGRAM.

\section{Judgment and Analysis of Seat Image}

Seat defects after image segmentation image clearly in the figure 8 shows the defect area and the surrounding of different texture. And after clustering on the gray-level histogram can discern gray level 118 pixels appears the frequency highest, and healthy regional gray levels of pixels to an average of 125 . Visible, seat surface defects after the clustering growth area is in the health area present low pixels, judging on the basis of measured the seat surface of the skin defects, then can eliminate the unqualified seat products from production line. Table 1 for the defect area and compares the image segmentation health area.

TABLE I. DEFECT DETECTION OF SEAT.

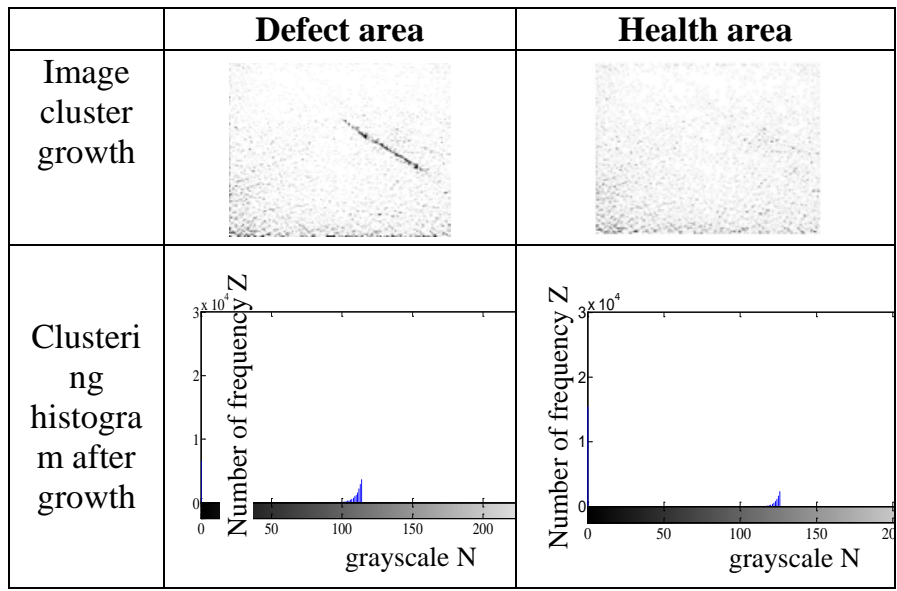

Can be seen from table 1 in reduced texture background conditions, and after clustering segmentation of defect features can be extracted, and compared with health area of grey value, known defect area a small seven grayscale health regions. Through region growing method, the method realized quality inspection on the surface of the seat. Through the defect area, the coordinates of grey value determined defect then statistical defect and the number of features, providing valuable data resources for quality improvement work.

\section{V.CONCLUSION}

The surface quality of automobile seat is inspected based on machine vision. The CCD acquisition images are pre-processed by graying and filtering to obtain better image quality. Then, the k-means clustering region growing method is performed for image segmentation in order to extract defect features. Experimental results show that the clustering growth defects after the average gray level image is 118 , which is distinctly different with healthy image grey value of 125 . It can 
determine the inspection scheme which can be finished in a short time. With the proposed methed, the detection effect is obvious and can greatly improve production efficiency.

\section{REFERENCE}

[1] C.C. Ho, M.C. Chen. Nighttime Fire Smoke Detection System Based on Machine Vision[J]. London: International Journal of Engineering and Manufacturing, 2012(13): 1369-1376.

[2] Subramanian Rajesh, De James St Germain H., Drake Samuel. Integrating a Vision System a Coordinate Measuring Machine to Automate the Datum Alignment Process[J]. American Society of Mechanical Engineers, 2005(6): 655 661.

[3] Al-Kindi Ghassan A., Shirinzadeh Bijan. Feasibility Assessment of Vision-based Surface Roughness Parameters Acquisition for Different Types of Machined Specimens[J]. Image and Vision Computing, 2009, 27(4): 444 458.

[4] J. Fu. Design of Automobile Heat Exchange Dimensional Measurement and Surface Inspection System Based on Machine Vision[D]. Nanjing: System Engineering College of Nanjing University of Science and Technology, 2014.

[5] Hendra Prima Syahputra, Tae Jo Ko. Application of Image Processing to Micro-milling Process for Surface Texturing[J]. International Journal of Precision Engineering and Manufacturing, 2013(14): 1507-1512.

[6] X.W. Chen, B. Xiao, Q. Gao. Defect Detection of Capacitor Aluminum Shell Surface Based on Machine Vision[D]. Jilin: Northeast Dianli University Institute of Mechanical Engineering, 2013.

[7] M.M.Abdelsamea. An Automatic Seeded Region Growing for 2D Biomedical Image Segmentation[A]. In: The proceedings of 2011 International Conference on Environment and BioScience [C]. Egypt: Assiut University, 2011.

[8] J. Wu, S. Poehlman, M. D. Noseworthy. Texture Feature based Automated Seeded Region Growing in Abdominal MRI Segmentation [A]. In: 2008 International Conference on Biomedical Engineering and Informatics [C]. Sanya, China: Scientific Research Publishing, 2008, 2730.

[9] J.Y. Wang, F. Zhang, X.Z. Zhou. Segmentation of Caption Region Using Wavelet Transform and Clustering[D]. Nanjing: School of Automation, Nanjing University of Science \&Technology, 2006. 